\title{
Infections and Chronic Inflammatory Bowel Disease
}

\author{
Torsten Kucharzik $^{\mathrm{a}}$ Christian Maaser ${ }^{\mathrm{b}}$ \\ ${ }^{a}$ Department of General Internal Medicine and Gastroenterology, University Teaching Hospital Lüneburg, Lüneburg, Germany, \\ ${ }^{b}$ Department of Geriatric Medicine, University Teaching Hospital Lüneburg, Lüneburg, Germany
}

\section{Keywords}

Immunosuppression - Infectious complications .

Anti-TNF · Cytomegalovirus · Clostridium difficile

\section{Summary}

In the more recent years since the introduction of antiTNF therapy, the treatment strategy in chronic inflammatory bowel disease has developed more towards an early intensive, often double immunosuppression. While this leads to an improved therapeutic success, this intensified therapy also increases the risk for side effects and especially for infectious complications. The early detection of this complication in the immunocompromised patient is often more difficult due to the potential broad spectrum of infectious agents, the often atypical presentation in conjunction with the immunosuppression as well as often similar symptoms regarding intestinal infectious complications common for a flare of the underlying disease. In the first part, this overview will discuss the broad spectrum of potential infectious complications, using pulmonary infections as an example and presenting an algorithm for detection and therapy. In the second part, common intestinal infectious complications will be discussed from diagnosis to therapy.

\section{Introduction}

While the current treatment strategy of immunosuppression in patients with active inflammatory bowel disease (IBD) leads to a remission or at least reduction of symptoms in most

\section{Schlüsselwörter \\ Immunsuppression · Infektiöse Komplikationen · Anti-TNF · Zytomegalievirus · Clostridium difficile}

\section{Zusammenfassung}

In den vergangenen Jahren hat sich unter anderem durch die Einführung der Anti-TNF-Therapie die Behandlungsstrategie bei chronisch entzündlichen Darmerkrankungen vermehrt zu einer bereits im früheren Stadium intensivierten, häufig doppelten immunsuppressiven Therapie entwickelt. Bei verbessertem Erfolg hat diese intensivere Therapie jedoch auch ein erhöhtes Nebenwirkungsrisiko und hierbei vor allem infektiöse Komplikationen zur Folge. Die Diagnosestellung einer infektiösen Komplikation ist dabei durch das breite potenzielle Keimspektrum, die unter Immunsuppression häufig nicht klassische Präsentation der Infektionssymptome, aber auch durch die bei intestinalen Komplikationen häufig ähnlichen Symptome vergleichbar einer zunehmenden Aktivität der zugrunde liegenden chronisch entzündlichen Darmerkrankung erschwert. Im ersten Abschnitt dieser Übersicht soll am Beispiel der möglichen pulmonalen infektiösen Komplikationen das breite Spektrum sowie ein Algorithmus zur Diagnostik dargestellt werden. Im zweiten Abschnitt wird auf häufige infektiöse intestinale Komplikationen von der Diagnostik bis zur Therapie eingegangen.

patients, this strategy also induces an increased risk for infections, intestinal as well as extraintestinal. In order to ensure a safe therapy, early detection of infectious complications and initiation of the treatment is crucial. 


\section{What Kind of Infections Can Potentially \\ Be Expected under Immunosuppression in \\ Patients with Inflammatory Bowel Disease?}

Various case reports, cohort evaluations as well as analyses of multicenter trials regarding infectious complications have been published. Toruner et al. [1], for example, identified 100 consecutive IBD patients with opportunistic infections. The infectious spectrum in his cohort included viral infections, e.g. herpes simplex virus (HSV), herpes zoster, and cytomegalovirus (CMV), bacterial infections, e.g. Escherichia coli, Mycobacterium marinum, and Streptococcus, as well as fungal infections, e.g. Histoplasma capsulatum and Cryptococcus neoformans [1]. These examples just give an idea of the broad spectrum one can expect as potential infectious complications. In addition to more or less rare infections, the risk for classical infections such as urinary tract infections, skin infections, or respiratory infections is also increased in the immunocompromised host.

As the spectrum is broad, this article will focus on two major areas, i.e. infectious respiratory complications and infections mimicking an acute flare of the underlying IBD.

\section{Infectious Respiratory Complications}

Starting with respiratory complications, the most prominent, though not the most common candidate for respiratory infections with the introduction of anti-TNF therapy has been the reactivation of latent tuberculosis, leading to severe infections with a lethal course in some of these patients [2].

With the awareness of these potential complications and the introduction of mandatory screening for latent tuberculosis prior to anti-TNF induction, this specific complication could be dramatically reduced, demonstrating the effectiveness of prevention. In most countries, these preventive measures include taking the history regarding potential exposures to open tuberculosis in the past, chest radiography, and an interferon-gamma release assay [3]. However, several issues need to be taken into account. For one, testing under immunosuppression such as thiopurines might not yield valid results due to unresponsive lymphocytes, and negative pulmonary imaging does not rule out latent or active tuberculosis. Furthermore, one has to keep in mind that an exclusion of tuberculosis before the beginning of treatment does not rule out a new infection with tuberculosis later in life. Therefore, retesting should always be considered in patients with signs of unknown infection, especially following potential exposure such as after travelling to countries with a high prevalence of tuberculosis [4]. One also has to keep in mind that when tuberculosis occurs in patients on anti-TNF therapy, for example, it is more commonly atypical (extrapulmonary in $<50 \%$, disseminated in $25 \%$ of cases), with intestinal tuberculosis mimicking active Crohn's disease, therefore making the correct diagnosis even more difficult $[3,5]$.
A further strategy to reduce pneumonia is to vaccinate. Guidelines such as from the European Crohn's and Colitis Organisation (ECCO) recommend vaccination against seasonal influenza on a yearly basis as well as pneumococcal vaccination according to national guidelines [3].

Immunocompromised patients presenting with respiratory symptoms should be carefully examined for pneumonia. In the case of classical signs for bacterial pneumonia, positive auscultation, and typical infiltrate in a chest radiograph, antibiotic treatment in these patients under immunosuppression should always cover Streptococcus pneumonia.

Patients with inflammatory disease on immunomodulator therapy with pneumonia should also be tested for Legionella pneumophila [3]. Testing can be done by screening a urine sample for L. pneumophila antigen, for example. The most common route of transmission is airborne from warm water supplies containing water that was not heated above $70^{\circ} \mathrm{C}$ as well as air conditioning and humidifiers. As there is no available vaccination, the best preventive measurements are maintenance of water systems and adequate heating of the warm water supply. Curative treatment consists of macrolide or fluoroquinolone antibiotics [6, 7].

A further pulmonary infection to be considered in patients under immunosuppression is an infection with Nocardia species, an aerobic Gram-positive, weakly acid-fast actinomycete $[8,9]$, especially in patients receiving anti-TNF and particularly when also being treated with corticosteroids [3]. Nocardia is found worldwide in soil and can lead to pulmonary infections through inhalation, with hematogenous dissemination to the brain in up to $33 \%$ [8]. Screening for infection requires examination of sputum, pleural or bronchial lavage fluid by Gram stain, and a modified acid-fast stain. Antibiotic treatment consists of a continuous treatment with sulfamethoxazole/trimethoprim and/or ceftriaxone until the disappearance of all lesions, which can take month [8].

In addition to bacterial-caused pneumonia, patients under immunosuppression appear to be also at risk for parasitic as well as fungal infection, though the knowledge and recommendations regarding IBD patients are mainly based on case reports and smaller case series. As these pulmonary infections often present with atypical symptoms and cannot be detected purely by clinical examination and standard chest radiography in many cases, suspicion should always be raised in immunosuppressed IBD patients complaining about breathlessness, cough, and reduced strength leading to a low threshold for performing CT scans and bronchoscopy with bronchoalveolar lavages (BAL). The screening should then include Mycobacteria, Strongyloides stercoralis, Nocardia, Histoplasma, Cryptococcus sp., Aspergillus sp., and Pneumocystis jirovecii. The diagnosis of $P$. jirovecii, an atypical fungus, is based on identification in bronchopulmonary secretions or BAL fluid, and the gold standard for Candida infection is a positive culture from normally sterile body sites, meaning that cultures from other sites have to be interpreted in the clinical context as cultures 


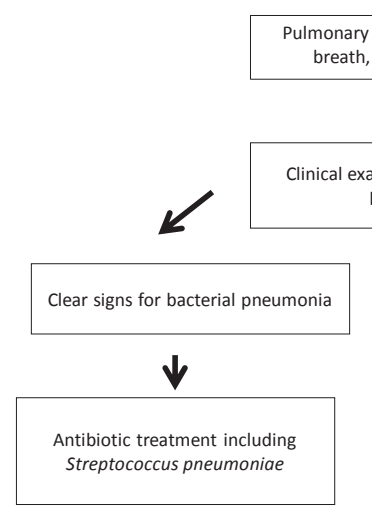

ptoms : cough, short of eath, fever, reduced strength

chest radiograph blood gas analysis

Fig. 1. Algorithm for immunocompromised IBD patients with pulmonary symptoms.

cannot differentiate colonization from infection in these cases. However, the sensitivity for e.g. Aspergillus sp. in BAL is only $50 \%$ and a definitive diagnosis may require invasive procedures such as biopsies. Similarly, the sensitivity for detection of Histoplasma capsulatum is only $50 \%$ [3]. Though the risk for these infections appears to be increased under immunosuppression, the actual number of IBD patients affected is fortunately relatively low. This implicates, however, that the individual experience of the treating gastroenterologist/surgeon is usually relatively low. Therefore, in unclear cases one should always consider consulting an infectious disease specialist for expert opinion, where available. The same holds true for the treatment of more rare infectious complications. The first-line treatment of $P$. jirovecii, for example, comprises the application of trimethoprim plus sulfamethoxazole for about 3 weeks, which requires monitoring of the blood count and the renal functions as this treatment may cause bone marrow suppression or renal impairment requiring dose adjustment [3].

While there are no effective immunizations available for the infectious agents discussed above and screening for these is not recommended in asymptomatic patients prior to initiation of immunosuppression, chemoprophylaxis for $P$. jirovecii is a potential option, at least for some patients. The ECCO guidelines as well as the German Colitis guideline recommend a standard prophylaxis with co-trimoxazole, if tolerated, for those patients on triple immunomodulators with one being a calcineurin inhibitor or anti-TNF therapy $[3,10]$. However, this recommendation is mainly based on expert opinion as risk-benefit studies for IBD patients are lacking, and therefore recommendations have to be extrapolated from patients with HIV infection and hemato-oncological diseases.

As discussed below in more detail for CMV, the potential spectrum for pulmonary infections also includes viruses such as from the family of herpes viruses, HSV, varicella zoster virus, Epstein-Barr virus, and CMV. Primary infection with e.g. HSV in immunocompetent individuals usually causes an asymptomatic or mild, self-limited oral-labial (HSV type 1) or genital (HSV type 2) infection [11]. In immunocompromised patients, especially in those treated with azathioprine, HSV infection has a greater potential for dissemination possibly causing severe systemic infections with significant morbidity and mortality including encephalitis, meningitis, pneumonia, esophagitis, colitis, and/or hepatitis [12-15]. While increasing titers of anti-HSV IgM (immunoglobulin M) can help making the diagnosis, the diagnostic gold standard is the PCR (polymerase chain reaction) from affected tissue [11]. Systemic therapy with the nucleoside analogue aciclovir is then required in symptomatic immunocompromised IBD patients [3, 11].

An algorithm for immunocompromised IBD patients with pulmonary symptoms is shown in figure 1 .

\section{Infections Mimicking a Flare}

\section{Clostridium difficile}

\section{Epidemiology}

In the past few years, the incidence of Clostridium difficile infection (CDI) has increased rapidly, with an infection rate that has doubled from 1996 to 2003 [16]. The burden of CDI has increased dramatically, and it is now recognized that CDI is responsible for $20-30 \%$ of cases of antibiotic-associated diarrhea and up to $75 \%$ of cases of antibiotic-associated colitis $[17,18]$. IBD has been found to be associated with $C$. difficile, and patients with IBD appear to be at an increased risk of developing CDI with a poorer outcome of CDI including higher rates of colectomy and death as well as higher rates of recurrence [19-22]. According to the recent studies, it appears that $\mathrm{CDI}$ is more common in patients with ulcerative colitis (UC) compared to Crohn's disease patients where an increase of CDI cases was only found in patients with large bowel disease [23]. In one study, CDI rates in UC patients have doubled from 2.66 to $5.12 \%$ in 7 years [24]. In addition to the increased prevalence, the number of IBD hospitalizations complicated by CDI has increased from $1.4 \%$ in 1998 to $2.9 \%$ in 2007 [25]. It has to be noted that $C$. difficile in IBD patients may not only affect the large bowel but also the small bowel, with high mortality rates which had frequently been shown in this population during the last decades [26]. CDI is often seen in patients who underwent proctocolectomy for a severe IBD [27] and also in patients with $C$. difficile pouchitis with CDI involvement in up to $18 \%$ of cases [28].

\section{Risk Factors}

Risk factors associated with CDI in the general population are antibiotic use, older age, hospitalization, immunosuppression, residence in long-term care facilities, cancer, and gastrointestinal disorders. IBD has been found to be an independent risk factor for CDI with a threefold increase compared with non-IBD patients [29]. There are conflicting data whether other factors associated with IBD including immunosuppression also play a role. It was found that glucocorticoid initiation with or without use of other immunosuppressive agents 
Table 1. Treatment of CID infection in IBD (according to IDSA guidelines [36])

\begin{tabular}{ll}
\hline Disease category & Treatment \\
\hline $\begin{array}{c}\text { Mild-to-moderate disease }- \\
\text { initial episode }\end{array}$ & $\begin{array}{l}\text { metronidazole } 500 \mathrm{mg} \text { three } \\
\text { times/day for } 10-14 \text { days }\end{array}$ \\
$\begin{array}{c}\text { Severe disease, uncomplicated - } \\
\text { initial episode }\end{array}$ & $\begin{array}{l}\text { vancomycin } 125 \mathrm{mg} \text { four times/ } \\
\text { day for } 10-14 \text { days }\end{array}$ \\
Severe disease, complicated - & vancomycin $500 \mathrm{mg}$ four times/ \\
initial episode & day plus metronidazole 500 mg \\
& every 8 h i.v. \\
Recurrence & same as for initial episode; \\
& alternative options: rifaximin, \\
fidaxomicin, fecal stool trans- & plantation \\
\hline
\end{tabular}

tripled the risk of CDI in IBD patients compared with the risk of other immunosuppressive agents [30]. Whether other immunomodulating drugs are associated with an increased risk of CDI infections is not clear. In a single study, an odds ratio of 2.56 for UC patients on maintenance immunosuppression was determined [22]. Other studies did not find an association between immunomodulation and the risk of CDI which supports the need for more intense research in this area [31]. Thus far, there is no evidence that biological agents such as infliximab or adalimumab are associated with an increased risk of CDI [24].

The role of antibiotics as a risk factor for CDI in IBD patients is also controversial. Antibiotic use alters normal bacterial flora and can lead to CDI. Some authors reported an increased use of antibiotics during the last couple of months prior to CDI $[22,30,32]$. In contrast, results from other studies have suggested that antibiotics may not be a risk factor for CDI in IBD [33, 34]. It is worth mentioning that the absence of antibiotic use should not lower the suspicion of CDI in IBD patients.

\section{Clinical Characteristics/Diagnosis}

It is extremely difficult to distinguish between symptoms of CDI and a flare of IBD. It is therefore important to always think of CDI in patients with IBD. There may also be atypical features of $C$. difficile in IBD in comparison to patients without IBD, e.g. frequent bloody stools or even absence of diarrhea $[26,29]$. It is worth mentioning that typical findings of CDI during endoscopy, such as pseudomembranous exudates which are typically found in patients with CDI, are often absent in patients with IBD in up to $87 \%$ of cases [35]. This should also be kept in mind if IBD patients undergo colonoscopy. The absence of pseudomembranes in a patient with IBD does therefore not exclude CDI.

CDI can be detected by common toxin assays and cultures. However, it should be noted that the commonly used enzyme immunoassays for toxin A and B have less sensitivity and specificity in comparison to nucleic acid amplification assays.
Current recommendations on CDI diagnosis implement a molecular assay or a two-step algorithm encompassing screening with enzyme immunoassay for glutamate dehydrogenase (GDH) followed by an enzyme immunoassay for toxins [36]. There is no evidence that testing for CDI should be done differently in IBD patients. On behalf of the physician, a high level of suspicion for CDI in IBD patients is required, and stool tests should be performed in every patient with IBD relapse. It should be noted that testing for CDI in non-IBD patients requires 1-2 probes; however, repeated testing up to 3 probes in IBD increases sensitivity.

\section{Treatment}

Even though local host defenses could be compromised in IBD due to the altered gut microflora or the use of immunomodulators, treatment of CDI in IBD patients is not fundamentally different compared to non-IBD patients. Currently, there are no clear guidelines regarding the treatment of CDI in IBD patients. Most authors suggest that the initial episode of CDI in IBD patients with mild-to-moderate disease scores should be treated with metronidazole (table 1). Patients with severe or complicated disease should be treated with vancomycin during the initial episode. C. difficile resistant to metronidazole has generally been uncommon in IBD patients even though failure rates of up to $50 \%$ have been reported in this population $[37,38]$. There is also no supporting evidence how to proceed with the ongoing immunosuppression in IBD patients with CDI. Most authors maintain immunosuppression in patients with CDI although an escalation of immunosuppression is avoided [39]. Severe CDI disease is usually treated in combination with vancomycin orally plus metronidazole i.v. In addition to metronidazole and vancomycin, other antibiotics like rifaximin or fidaxomicin may be used in severe or recurrent CDI. Beside antibiotics, fecal microbiota transplantation may be another option to treat concurrent or particularly recurrent CDI in IBD patients. The rationale behind fecal microbiota transplantation comes from the notion that an introduction of feces from the healthy host would recolonize the bowel with microbiota needed to reestablish colonization resistance against $C$. difficile. Even though there is no general protocol for the administration of fecal microbiota transplantation, several attempts have been made to treat CDI in IBD patients with more or less reasonable results [40]. There is currently no evident role of probiotics in the prevention or treatment of CDI in IBD patients. The treatment of recurrent CDI is even more complicated, and conflicting data exist on how to treat in this situation. Options include the use of the same treatment regimen as for the initial episode up to the use of alternative antibiotics such as rifaximin, fidaxomicin, or fecal microbiota transplantation in this situation [41].

In summary, CDI in IBD patients is a major challenge as the clinical importance is still underestimated. Because of the high mortality and severity of CDI in patients with IBD, early recognition in these patients is of particular importance. 


\section{Cytomegalovirus Infection}

The impact of CMV in IBD remains controversial [42]. The pathogenicity of CMV reactivation in IBD patients has been a debate in the literature during the last decade. However, despite arguments on the non-pathogenicity of CMV reactivation, there is overall agreement that IBD patients are at an increased risk of $\mathrm{CMV}$ reactivation and that failure to ensure the appropriate diagnosis and treatment may lead to significant morbidity and mortality. Subclinical reactivation of latent CMV infection may occur frequently and is often associated with immunomodulator therapy $[42,43]$. Usually, the reactivation of latent CMV is asymptomatic and does not induce serious tissue damage. It is therefore required to distinguish between CMV infection which may be detected by CMV DNA and CMV disease which may cause organ damage in the colon, liver, lung, or other organs [44]. Mild CMV reactivation without tissue damage is nearly always self-limited even under continuation of immunomodulator or biological treatment [4547]. Therefore, a stop of immunomodulator treatment in patients with CMV reactivation is usually not required.

\section{Clinical Presentation/Diagnosis}

Screening for CMV infection in all IBD patients is not indicated unless the patients are steroid-resistant. In patients with acute steroid-resistant colitis, CMV should be excluded [44]. Different techniques are used to diagnose CMV infection including endoscopy, histology, serology, as well as CMV antigen and CMV DNA testing. The use of the different techniques is controversial, and there is no international gold standard for the detection of CMV infection. The use of CMV serology is of limited value for diagnosing an acute infection because of its failure to detect CMV reactivation. However, CMV serology may identify patients who are CMV-IgG-positive and thus at risk for CMV reactivation [48]. CMV antigen and DNA testing may act as reliable markers for disseminated infection. Even though the antigenemia assays are only semiquantitative, they may act as indirect markers and are sufficient to monitor infection as well as antiviral treatment in the immunocompromised host. More sensitive is the detection of DNA viral load by PCR [49]. The advantages of qualitative and quantitative testing as well as of rapid and high sensitive results favor the detection of CMV DNA load by means of PCR in the blood or in tissue biopsies $[49,50]$. In steroid-refractory colitis, CMV has been detected by PCR in up to $36 \%$ of patients [51]. Detection of CMV DNA viral load of more than 250 copies/mg may be used as a predictor of steroid-resistant disease [52]. An alternative for the detection of CMV antigen or CMV DNA viral load may be the use of histopathology combined with immunohistochemistry. Monoclonal antibodies against CMV early antigen are highly specific and sensitive for verifying CMV infection in tissue biopsies [53]. Therefore, CMV is most commonly excluded by tissue PCR or immunohistochemistry [44].

\section{Treatment of CMV Colitis}

In the case of severe steroid-resistant colitis with CMV detected in the mucosa during immunomodulator therapy, antiviral therapy should be initiated and immunomodulators considered to be discontinued until colitis symptoms improve [44]. The therapy of choice for CMV infections is ganciclovir for a period of 2-3 weeks [54]. After 3-5 days a switch to oral valganciclovir for the rest of the period of 2-3 weeks may be considered $[49,55,56]$. In patients where ganciclovir is associated with side effects such as myelotoxicity or in those with ganciclovir resistance, foscarnet for a period of 2-3 weeks may be an alternative $[42,57]$. Subclinical CMV reactivation or mild CMV infection does not require antiviral treatment or interruption of immunomodulator therapy as it usually completely recovers [54]. In the case of CMV disease with CMV reactivation causing organ disease such as hepatitis, pneumonia, esophagitis, colitis, or meningoencephalitis, immediate antiviral treatment with ganciclovir and discontinuation of immunosuppression is usually required due to the poor outcome in this situation $[55,58,59]$.

All in all, screening for CMV infection is usually not required in IBD patients before immunomodulator therapy has been started. In patients with acute steroid-resistant colitis or in patients with acute flares under steroid treatment, CMV infection should be excluded either by PCR or immunohistochemistry. According to the recent ECCO guidelines, patients with severe steroid-resistant colitis with CMV infection during immunomodulator therapy should be treated with antiviral drugs until colitis symptoms improve. In the case of CMV disease with systemic organ manifestation, immunomodulator therapy should be discontinued.

In summary, there is a broad spectrum of potential infectious complications in immunocompromised patients. Some infections mimic a flare of the underlying chronic bowel disease, while others present in an atypical fashion due to the immunosuppression. This demonstrates the necessity for carefully taking the patients' history especially regarding new symptoms. Furthermore, patients should clearly be advised to report new symptoms to the treating physician. Carrying a document listing the taken immunosuppression, which can be presented to the emergency room team in the case of urgent hospital admissions, might contribute to infectious signs not being underestimated in the often young IBD patients.

\section{Disclosure Statement}

Torsten Kucharzik: Consultation fee or speaker's honoraria from AbbVie, MSD, UCB, Falk Foundation, Ferring, Shire, and Wolff Pharma.

Christian Maaser: Consultation fee or speaker's honoraria from AbbVie, MSD, UCB, Falk Foundation, Ferring, Shire, and Wolff Pharma. 


\section{References}

1 Toruner M, Loftus EV Jr, Harmsen WS, et al: Risk factors for opportunistic infections in patients with inflammatory bowel disease. Gastroenterology 2008;134:929-936.

2 Cantini F, Niccoli L, Goletti D: Adalimumab, etanercept, infliximab, and the risk of tuberculosis: data from clinical trials, national registries, and postmarketing surveillance. J Rheumatol Suppl 2014;91:47-55.

3 Rahier JF, Magro F, Abreu C, et al: Second European evidence-based consensus on the prevention, diagnosis and management of opportunistic infections in inflammatory bowel disease. J Crohns Colitis 2014;8:443-468.

4 Abreu C, Magro F, Santos-Antunes J, et al: Tuberculosis in anti-TNF-alpha treated patients remains a problem in countries with an intermediate incidence: analysis of 25 patients matched with a control population. J Crohns Colitis 2013; 7:e486-492.

5 Bruzzese V, Lorenzetti R, Zullo A, et al: Intestina tuberculosis mimicking Crohn disease in infliximab-treated rheumatoid arthritis. J Clin Rheumatol 2012;18:324.

6 Diederen BM: Legionella spp. and Legionnaires disease. J Infect 2008;56:1-12.

7 Lanternier F, Tubach F, Ravaud P, et al: Incidence and risk factors of Legionella pneumophila pneumonia during anti-tumor necrosis factor therapy: a prospective French study. Chest 2013;144:990-998.

8 Yildiz O, Doganay M: Actinomycoses and Nocardia pulmonary infections. Curr Opin Pulm Med 2006;12:228-234.

$\checkmark 9$ Ford AC, Peyrin-Biroulet L: Opportunistic infections with anti-tumor necrosis factor-alpha therapy in inflammatory bowel disease: meta-analysis of randomized controlled trials. Am J Gastroenterol 2013;108:1268-1276.

10 Dignass A, Preiss JC, Aust DE, et al: Updated German guideline on diagnosis and treatment of ulcerative colitis, 2011 (article in German). Z Gastroenterol 2011;49:1276-1341.

11 Fatahzadeh M, Schwartz RA: Human herpes simplex virus infections: epidemiology, pathogenesis, symptomatology, diagnosis, and management. J Am Acad Dermatol 2007;57:737-763; quiz 764766.

12 Cottone M, Renna S: IBD: incidence of HSV and HPV with azathioprine. Nat Rev Gastroenterol Hepatol 2009;6:444-445.

13 Seksik P, Cosnes J, Sokol H, et al: Incidence of benign upper respiratory tract infections, HSV and HPV cutaneous infections in inflammatory bowel disease patients treated with azathioprine. Aliment Pharmacol Ther 2009;29:1106-1113.

14 el-Serag HB, Zwas FR, Cirillo NW, et al: Fulminant herpes colitis in a patient with Crohn's disease. J Clin Gastroenterol 1996;22:220-223.

$\checkmark 15$ Shlien RD, Meyers S, Lee JA, et al: Fulminant herpes simplex hepatitis in a patient with ulcerative colitis. Gut 1988;29:257-261.

16 McDonald LC, Owings M, Jernigan DB: Clostridium difficile infection in patients discharged from US short-stay hospitals, 1996-2003. Emerg Infect Dis 2006;12:409-415.

17 Bartlett JG: Clostridium difficile: clinical considerations. Rev Infect Dis 1990;12(suppl 2):S243-251.

18 Bartlett JG: Narrative review: the new epidemic of Clostridium difficile-associated enteric disease. Ann Intern Med 2006;145:758-764.
Bien J, Palagani V, Bozko P: The intestinal microbiota dysbiosis and Clostridium difficile infection: is there a relationship with inflammatory bowel disease? Therap Adv Gastroenterol 2013;6:53-68.

20 Sinh P, Barrett TA, Yun L: Clostridium difficile infection and inflammatory bowel disease: a review. Gastroenterol Res Pract 2011;2011:136064.

21 Navaneethan U, Venkatesh PG, Shen B: Clostridium difficile infection and inflammatory bowel disease: understanding the evolving relationship. World J Gastroenterol 2010;16:4892-4904.

22 Issa M, Vijayapal A, Graham MB, et al: Impact of Clostridium difficile on inflammatory bowel disease. Clin Gastroenterol Hepatol 2007;5:345-351.

23 Ricciardi R, Ogilvie JW Jr, Roberts PL, et al: Epidemiology of Clostridium difficile colitis in hospitalized patients with inflammatory bowel diseases. Dis Colon Rectum 2009;52:40-45.

24 Nguyen GC, Kaplan GG, Harris ML, et al: A national survey of the prevalence and impact of Clostridium difficile infection among hospitalized inflammatory bowel disease patients. Am J Gastroenterol 2008;103:1443-1450.

25 Ananthakrishnan AN, McGinley EL, Binion DG: Excess hospitalisation burden associated with Clostridium difficile in patients with inflammatory bowel disease. Gut 2008;57:205-210.

26 Issa M, Ananthakrishnan AN, Binion DG: Clostridium difficile and inflammatory bowel disease. Inflamm Bowel Dis 2008;14:1432-1442.

27 Blossom DB, McDonald LC: The challenges posed by reemerging Clostridium difficile infection. Clin Infect Dis 2007;45:222-227.

28 Shen BO, Jiang ZD, Fazio VW, et al: Clostridium difficile infection in patients with ileal pouch-anal anastomosis. Clin Gastroenterol Hepatol 2008; 6: 782-788.

29 Rodemann JF, Dubberke ER, Reske KA, et al: Incidence of Clostridium difficile infection in inflammatory bowel disease. Clin Gastroenterol Hepatol 2007;5:339-344.

30 Schneeweiss S, Korzenik J, Solomon DH, et al: Infliximab and other immunomodulating drugs in patients with inflammatory bowel disease and the risk of serious bacterial infections. Aliment Pharmacol Ther 2009;30:253-264.

31 Kariv R, Navaneethan U, Venkatesh PG, et al: Impact of Clostridium difficile infection in patients with ulcerative colitis. J Crohns Colitis 2011;5:34-40.

32 Meyer AM, Ramzan NN, Loftus EV Jr, et al: The diagnostic yield of stool pathogen studies during relapses of inflammatory bowel disease. J Clin Gastroenterol 2004;38:772-775.

33 Bossuyt P, Verhaegen J, Van Assche G, et al: Increasing incidence of Clostridium difficile-associated diarrhea in inflammatory bowel disease. J Crohns Colitis 2009;3:4-7.

34 Kelsen JR, Kim J, Latta D, et al: Recurrence rate of clostridium difficile infection in hospitalized pediatric patients with inflammatory bowel disease. Inflamm Bowel Dis 2011;17:50-55.

35 Ben-Horin S, Margalit M, Bossuyt P, et al: Prevalence and clinical impact of endoscopic pseudomembranes in patients with inflammatory bowel disease and Clostridium difficile infection. J Crohns Colitis 2010;4:194-198.

36 Cohen SH, Gerding DN, Johnson S, et al: Clinical practice guidelines for Clostridium difficile infection in adults: 2010 update by the Society for Healthcare Epidemiology of America (SHEA) and the Infectious Diseases Society of America (IDSA). Infect Control Hosp Epidemiol 2010;31:431-455.
7 Mazmanian SK, Liu CH, Tzianabos AO, et al: An immunomodulatory molecule of symbiotic bacteria directs maturation of the host immune system. Cell 2005;122:107-118.

38 Koo HL, Koo DC, Musher DM, et al: Antimotility agents for the treatment of Clostridium difficile diarrhea and colitis. Clin Infect Dis 2009;48:598-605.

39 Surawicz CM, Brandt LJ, Binion DG, et al: Guidelines for diagnosis, treatment, and prevention of Clostridium difficile infections. Am J Gastroenterol 2013;108:478-498; quiz 499

40 Brandt LJ, Aroniadis OC, Mellow M, et al: Longterm follow-up of colonoscopic fecal microbiota transplant for recurrent Clostridium difficile infection. Am J Gastroenterol 2012;107:1079-1087.

41 Reddy SS, Brandt LJ: Clostridium difficile infection and inflammatory bowel disease. J Clin Gastroenterol 2013;47:666-671.

42 Kandiel A, Lashner B: Cytomegalovirus colitis complicating inflammatory bowel disease. Am J Gastroenterol 2006;101:2857-2865.

43 Matsuoka K, Iwao Y, Mori T, et al: Cytomegalovirus is frequently reactivated and disappears without antiviral agents in ulcerative colitis patients. Am J Gastroenterol 2007:102:331-337.

44 Rahier JF, Magro F, Abreu C, et al; European Crohn's and Colitis Organisation (ECCO): Second European evidence-based consensus on the prevention, diagnosis and management of opportunistic infections in inflammatory bowel disease. $\mathrm{J}$ Crohns Colitis 2014;8:443-468.

45 Lavagna A, Bergallo M, Daperno M, et al: Inflixi$\mathrm{mab}$ and the risk of latent viruses reactivation in active Crohn's disease. Inflamm Bowel Dis 2007; 13:896-902.

46 Nakase H, Chiba T: TNF-alpha is an important pathogenic factor contributing to reactivation of cytomegalovirus in inflamed mucosa of colon in patients with ulcerative colitis: lesson from clinical experience. Inflamm Bowel Dis 2010;16:550-551.

47 Criscuoli V, Mocciaro F, Orlando A, et al: Cytomegalovirus disappearance after treatment for refractory ulcerative colitis in 2 patients treated with infliximab and 1 patient with leukapheresis. Inflamm Bowel Dis 2009;15:810-811.

48 Aulitzky WE, Hengster P, Tilg H, et al: Cytomegalovirus infection. Wien Klin Wochenschr 1988; 100:33-43.

49 Gerna G, Zavattoni M, Percivalle E, et al: Diagnosis of human cytomegalovirus infections in the immunocompromised host. Clin Diagn Virol 1996;5: 181-186.

50 de Jong MD, Galasso GJ, Gazzard B, et al: Summary of the II International Symposium on Cytomegalovirus. Antiviral Res 1998;39:141-162.

51 Papadakis KA, Tung JK, Binder SW, et al: Outcome of cytomegalovirus infections in patients with inflammatory bowel disease. Am J Gastroenterol 2001;96:2137-2142.

52 Roblin X, Pillet S, Oussalah A, et al: Cytomegalovirus load in inflamed intestinal tissue is predictive of resistance to immunosuppressive therapy in ulcerative colitis. Am J Gastroenterol 2011;106:20012008

53 Mills AM, Guo FP, Copland AP, et al: A comparison of CMV detection in gastrointestinal mucosal biopsies using immunohistochemistry and PCR performed on formalin-fixed, paraffin-embedded tissue. Am J Surg Pathol 2013;37:995-1000. 
54 Rowshani AT, Bemelman FJ, van Leeuwen EM, et al: Clinical and immunologic aspects of cytomegalovirus infection in solid organ transplant recipients. Transplantation 2005;79:381-386.

55 Kim YS, Kim YH, Kim JS, et al: The prevalence and efficacy of ganciclovir on steroid-refractory ulcerative colitis with cytomegalovirus infection: a prospective multicenter study. J Clin Gastroenterol 2012;46:51-56.
56 Cottone M, Pietrosi G, Martorana G, et al: Prevalence of cytomegalovirus infection in severe refractory ulcerative and Crohn's colitis. Am J Gastroenterol 2001;96:773-775.

57 Shimada Y, Iiai T, Okamoto H, et al: Toxic megacolon associated with cytomegalovirus infection in ulcerative colitis. J Gastroenterol 2003:38:11071108.
58 Kim JJ, Simpson N, Klipfel N, et al: Cytomegalovirus infection in patients with active inflammatory bowel disease. Dig Dis Sci 2010;55:1059-1065.

59 Dimitroulia E, Spanakis N, Konstantinidou AE, et al: Frequent detection of cytomegalovirus in the intestine of patients with inflammatory bowel disease. Inflamm Bowel Dis 2006;12:879-884. 